MATHEMATICS OF COMPUTATION

Volume 70, Number 233 , Pages 17-25

S $0025-5718(99) 01180-1$

Article electronically published on August 17, 1999

\title{
A TWO-GRID DISCRETIZATION SCHEME FOR EIGENVALUE PROBLEMS
}

\author{
JINCHAO XU AND AIHUI ZHOU
}

\begin{abstract}
A two-grid discretization scheme is proposed for solving eigenvalue problems, including both partial differential equations and integral equations. With this new scheme, the solution of an eigenvalue problem on a fine grid is reduced to the solution of an eigenvalue problem on a much coarser grid, and the solution of a linear algebraic system on the fine grid and the resulting solution still maintains an asymptotically optimal accuracy.
\end{abstract}

\section{INTRODUCTION}

The purpose of this paper is to present some discretization techniques based on two finite element spaces for solving eigenvalue problems, including both partial differential equations and integral equations.

The two-grid method used for discretization was first introduced by $\mathrm{Xu}[13,15$. 16] for nonsymmertic and nonlinear elliptic problems. Later on, it was further investigated by many other authors, for instance, Axelsson and Layton [2] for nonlinear elliptic problems, Dawson and Wheeler [7] and Dawson, Wheeler and Woodward 8] for finite difference scheme for parabolic equations, Layton and Lenferink [9] and Utnes [12 for Navier-Stokes equations, Marion and Xu 11 for evolution equations, and $\mathrm{Xu}$ and Zhou 17 for parallelization of two-grid discretizations.

In this paper we propose a two-grid discretization for eigenvalue problems. With the new proposed scheme, for example, solving an elliptic eigenvalue problem will not be much more difficult than the solution of some standard elliptic boundary value problem. A similar scheme can also be applied to solve integral eigenvalue problems. Our method is an iterative method, which is, in a way, related to that in Lin [10]. The method in this paper, however, is based on two finite element spaces with different scales.

The standard Galerkin approximation scheme for eigenvalue problems has been extensively investigated, see e.g., Babuska and Osborn [3, 4], Chatelin [5], and references cited therein, and some basic results in these papers are employed in our discussions.

Received by the editor December 16, 1998 and, in revised form, February 25, 1999.

2000 Mathematics Subject Classification. Primary 65L15, 65N15, 65N25, 65N30, 65 N55.

Key words and phrases. Eigenvalue problems, finite elements, partial differential equations, integral equations, two-grid method.

This work was partially supported by NSF DMS-9706949, NSF ACI-9800244 and NASA NAG21236 through Penn State, and the Center for Computational Mathematics and Applications, The Pennsylvania State University, and by NSF ASC 9720257 through UCLA. The second author was also partially supported by National Science Foundation of China.

(C)1999 American Mathematical Society 

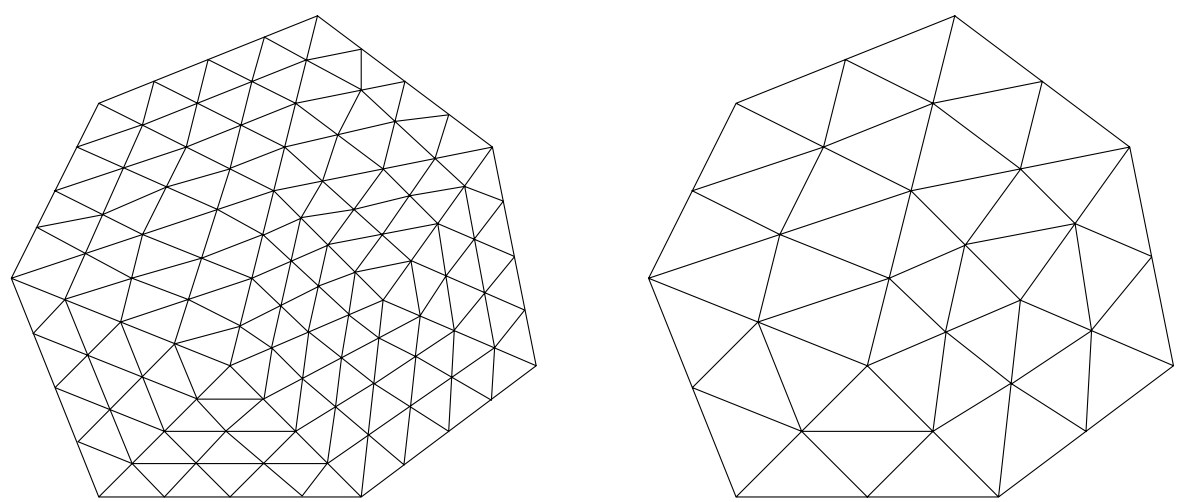

Figure 1. Two grids

In the remainder of this section, we would like to give a simple example to illustrate the main idea in this paper. Consider the following eigenvalue problem posed on a convex polygonal domain $\Omega \subset R^{2}$ :

$$
\begin{array}{rlr}
-\Delta u & =\lambda u, & \text { in } \Omega, \\
u & =0, & \text { on } \partial \Omega .
\end{array}
$$

Let $S^{H}(\Omega)$ and $S^{h}(\Omega)$, satisfying $S^{H}(\Omega) \subset S^{h}(\Omega) \subset H_{0}^{1}(\Omega)$, be two linear finite element subspaces associated with a coarse grid $T^{H}(\Omega)$ and the refined grid $T^{h}(\Omega)$ (see Figure 1), respectively. We can employ the following algorithm to approximate the problem (1.1), say the first eigenvalue $\lambda$ with its corresponding eigenvector $u$ and $\|\nabla u\|_{L^{2}}=1$ (see Sections 4 and 5):

1. Solve an eigenvalue problem on a coarse grid: Find $\lambda_{H} \in R^{1}, u_{H} \in S^{H}(\Omega)$ such that $\left\|\nabla u_{H}\right\|_{L^{2}}=1$ and

$$
\int_{\Omega} \nabla u_{H} \cdot \nabla v=\lambda_{H} \int_{\Omega} u_{H} v, \quad \forall v \in S^{H}(\Omega) .
$$

2. Solve one single linear problem on a fine grid: Find $u^{h} \in S^{h}(\Omega)$ such that

$$
\int_{\Omega} \nabla u^{h} \cdot \nabla v=\lambda_{H} \int_{\Omega} u_{H} v, \quad \forall v \in S^{h}(\Omega) .
$$

3. Compute the Rayleigh quotient

$$
\lambda^{h}=\frac{\left\|\nabla u^{h}\right\|_{L^{2}}^{2}}{\left\|u^{h}\right\|_{L^{2}}^{2}}
$$

If, for example, $\lambda_{H}$ is the first eigenvalue of the problem at the first step, then we can establish the following results (see Sections 4 and 5 )

$$
\left\|\nabla\left(u-u^{h}\right)\right\|_{L^{2}}=O\left(h+H^{2}\right) \text { and }\left|\lambda^{h}-\lambda\right|=O\left(h^{2}+H^{4}\right) .
$$

These estimates mean that we can obtain asymptotically optimal errors by taking $H=O(\sqrt{h})$. 


\section{Preliminaries}

In this section, we shall describe some basic notation and properties of the standard finite element approximation.

Throughout this paper, we shall use the letter C (with or without subscripts) to denote a generic positive constant which may stand for different values at its different occurrences. For convenience, following [14], the symbols $\lesssim, \gtrsim$ and $\equiv$ will be used in this paper. The expressions $x_{1} \lesssim y_{1}, x_{2} \gtrsim y_{2}$ and $x_{3} \underset{\sim}{\approx} y_{3}$, mean that $x_{1} \leq C_{1} y_{1}, x_{2} \geq c_{2} y_{2}$ and $c_{3} x_{3} \leq y_{3} \leq C_{3} x_{3}$ for some constants $C_{1}, c_{2}, c_{3}$ and $C_{3}$ that are independent of the mesh parameters (and the exact eigenvalues).

Suppose that $(X,\|\cdot\|)$ is a real Hilbert space with inner product $(\cdot, \cdot)$ and norm $\|\cdot\|$. Let $a(\cdot, \cdot), b(\cdot, \cdot)$ be two symmetric bilinear forms on $X \times X$ satisfying

$$
\begin{gathered}
a(w, v) \lesssim\|w\|\|v\|, \forall w, v \in X, \\
\|w\|^{2} \lesssim a(w, w), \forall w \in X \text { and } 0<b(w, w), \forall w \in X, w \neq 0 .
\end{gathered}
$$

We note that $\|\cdot\|_{a} \equiv a(\cdot, \cdot)^{1 / 2}$ and $\|\cdot\|$ are two equivalent norms on $X$. We assume that the norm $\|\cdot\|$ is relatively compact with respect to the norm

$$
\|w\|_{b} \equiv b(w, w)^{1 / 2}
$$

in the sense that from any sequence which is bounded in $\|\cdot\|$, one can extract a subsequence which is Cauchy with respect to $\|\cdot\|_{b}$. In the rest of this paper, we shall use $a(\cdot, \cdot)$ and $\|\cdot\|_{a}$ as the inner product and norm on $X$, and denote this space by $X_{a}$. Set

$$
X_{b}=\text { the completion of } X_{a} \text { with respect to }\|\cdot\|_{b} \text {. }
$$

Thus $X_{b}$ is a Hilbert space with inner product $b(\cdot, \cdot)$ and is compactly imbedded in $X_{a}$. Construct a "negative space" $X_{-a}=$ the dual of $X_{a}$ with a norm $\|\cdot\|_{-a}$ given by

$$
\|w\|_{-a}=\sup _{v \in X_{a},\|v\|_{a}=1} b(w, v) .
$$

Then $X_{b} \subset X_{-a}$ compactly, and for $v \in X_{a}, b(w, v)$ has a continuous extension to $w \in X_{-a}$ so that $b(w, v)$ is continuous on $X_{-a} \times X_{a}$.

We assume that $S^{h} \subset X_{a}$ is a family of finite-dimensional spaces that satisfy the following assumption: For any $w \in X_{a}$,

$$
\lim _{h \rightarrow 0} \inf _{v \in S^{h}}\|w-v\|_{a}=0
$$

Let $P_{h}$ be the orthogonal projection of $X_{a}$ onto $S^{h}$ defined by

$$
a\left(w-P_{h} w, v\right)=0, \forall w \in X_{a}, v \in S^{h} .
$$

Clearly,

$$
\left\|P_{h} w\right\|_{a} \leq\|w\|_{a}, \quad \forall w \in X_{a} .
$$

If $w \in X_{a}$, then, by (2.3)

$$
\left\|w-P_{h} w\right\|_{a}=o(1), \quad \text { as } h \rightarrow 0 .
$$

Let $\eta_{a}(h)$ be defined by

$$
\eta_{a}(h)=\sup _{f \in X_{a},\|f\|_{a}=1} \inf _{v \in S^{h}}\left\|L^{-1} f-v\right\|_{a},
$$


where $L^{-1}: X_{-a} \longrightarrow X_{a}$ satisfies

$$
a\left(L^{-1} f, v\right)=b(f, v), \forall f \in X_{-a}, v \in X_{a} .
$$

We have the following results (see Lemmas 3.3 and 3.4 in [3])

\section{Lemma 2.1.}

$$
\eta_{a}(h)=o(1) \text { as } h \rightarrow 0
$$

and

$$
\left\|w-P_{h} w\right\|_{-a} \lesssim \eta_{a}(h)\left\|w-P_{h} w\right\|_{a}, \quad \forall w \in X_{a} .
$$

\section{A STANDARD DiscRETIZATION}

A number $\lambda$ is called an eigenvalue of the form $a(\cdot, \cdot)$ relative to the form $b(\cdot, \cdot)$ if there is a nonzero vector $u \in X_{a}$, called an associated eigenvector, satisfying

$$
a(u, v)=\lambda b(u, v), \forall v \in X_{a} .
$$

It is known that (3.1) has a countable sequence of real eigenvalues

$$
0<\lambda_{1} \leq \lambda_{2} \leq \lambda_{3} \leq \cdots
$$

and corresponding eigenvectors

$$
u_{1}, u_{2}, u_{3}, \cdots
$$

which can be assumed to satisfy

$$
a\left(u_{i}, u_{j}\right)=\lambda_{j} b\left(u_{i}, u_{j}\right)=\delta_{i j} .
$$

In the sequence $\left\{\lambda_{j}\right\}$, the $\lambda_{j}$ are repeated according to geometric multiplicity.

A standard finite element scheme for (3.1) is: Find a pair $\left(\lambda_{h}, u_{h}\right)$, where $\lambda_{h}$ is a number and $0 \neq u_{h} \in S^{h}$, satisfying

$$
a\left(u_{h}, v\right)=\lambda_{h} b\left(u_{h}, v\right), \forall v \in S^{h},
$$

and use $\lambda_{h}$ and $u_{h}$ as approximations to $\lambda$ and $u$ (as $h \rightarrow 0$ ), respectively. One sees that (3.2) has a finite sequence of eigenvalues

$$
0<\lambda_{1, h} \leq \lambda_{2, h} \leq \cdots \leq \lambda_{n_{h}, h}, n_{h}=\operatorname{dim} S^{h},
$$

and corresponding eigenvectors

$$
u_{1, h}, u_{2, h}, \cdots, u_{n_{h}, h}
$$

which can be assumed to satisfy

$$
a\left(u_{i, h}, u_{j, h}\right)=\lambda_{j, h} b\left(u_{i, h}, u_{j, h}\right)=\delta_{i j} .
$$

It follows directly from the minimum-maximum principle (see [4] or [5]) that

$$
\lambda_{i} \leq \lambda_{i, h}, i=1,2, \cdots, n_{h} .
$$

Set

$$
\delta_{h}\left(\lambda_{i}\right)=\sup _{w \in M\left(\lambda_{i}\right)} \inf _{v \in S^{h}}\|w-v\|_{a}
$$

and

$$
M\left(\lambda_{i}\right)=\left\{w \in X_{a}: w \text { is an eigenvector of (3.1) corresponding to } \lambda_{i},\|w\|_{a}=1\right\} .
$$

The following results (see p. 699 of [4] and Lemma 3.7 and (3.29b) of [3], or cf. [5]) will be employed in the coming discussions. 
Proposition 3.1. (i) For any $u_{i, h}$ of (3.2) $\left(i=1,2, \cdots, n_{h}\right)$, there is an eigenvalue $u^{i}$ of (3.1) corresponding to $\lambda_{i}$ satisfying $\left\|u^{i}\right\|_{a}=1$ and

$$
\left\|u^{i}-u_{i, h}\right\|_{a} \leq C_{i} \delta_{h}\left(\lambda_{i}\right) .
$$

Moreover,

$$
\left\|u^{i}-u_{i, h}\right\|_{-a} \leq C_{i} \eta_{a}(h)\left\|u^{i}-u_{i, h}\right\|_{a} .
$$

(ii) For eigenvalues,

$$
\lambda_{i} \leq \lambda_{i, h} \leq \lambda_{i}+C_{i} \delta_{h}^{2}\left(\lambda_{i}\right)
$$

Here and hereafter $C_{i}$ is some constant depending on $i$ but not depending on the mesh parameter $h$.

\section{A TWO-GRID DisCRETIZATION}

In this section, we propose a two-grid discretization for the eigenvalue problem. There is some superclose relationship between the Ritz-Galerkin projection of the eigenvector and the finite element approximation to the eigenvector:

\section{Proposition 4.1.}

$$
\left\|P_{h} u^{i}-u_{i, h}\right\|_{a} \lesssim \lambda_{i, h}-\lambda_{i}+\lambda_{i}\left\|u^{i}-u_{i, h}\right\|_{-a} .
$$

Proof. From the identity

$$
a\left(P_{h} u^{i}-u_{i, h}, v\right)=\left(\lambda_{i}-\lambda_{i, h}\right) b\left(u_{i, h}, v\right)+\lambda_{i} b\left(u^{i}-u_{i, h}, v\right), \forall v \in S^{h},
$$

we immediately obtain the result.

Our analysis is based on the following crucial (but straightforward) property of eigenvalue and eigenvector approximation (see e.g. Lemma 3.1 of [3] or Lemma 9.1 of [4]).

Proposition 4.2. Let $(\lambda, u)$ be an eigenvalue pair of (3.1). For any $w \in X_{a} \backslash\{0\}$,

$$
\frac{a(w, w)}{b(w, w)}-\lambda=\frac{a(w-u, w-u)}{b(w, w)}-\lambda \frac{b(w-u, w-u)}{b(w, w)} .
$$

Algorithm 4.1. 1. Find $\lambda_{i, H} \in R^{1}$ and $u_{i, H} \in S^{H}\left(i=1,2, \cdots, n_{H}\right)$ such that $\left\|u_{i, H}\right\|_{a}=1$ and

$$
a\left(u_{i, H}, v\right)=\lambda_{i, H} b\left(u_{i, H}, v\right), \forall v \in S^{H} ;
$$

2. Find $u^{i, h} \in S^{h}\left(i=1,2, \cdots, n_{H}\right)$ such that

$$
a\left(u^{i, h}, v\right)=\lambda_{i, H} b\left(u_{i, H}, v\right), \forall v \in S^{h} ;
$$

3. Set

$$
\lambda^{i, h}=\frac{a\left(u^{i, h}, u^{i, h}\right)}{b\left(u^{i, h}, u^{i, h}\right)}, i=1,2, \cdots, n_{H} .
$$

Theorem 4.3. Assume that $\left(\lambda^{i, h}, u^{i, h}\right)\left(i=1,2, \cdots, n_{H}\right)$ are obtained by Algorithm 4.1. If $S^{H} \subset S^{h}$, then

$$
\left\|u^{i}-u^{i, h}\right\|_{a} \lesssim \lambda_{i, H}-\lambda_{i}+\lambda_{i}\left\|u^{i}-u_{i, H}\right\|_{-a}+\left\|u^{i}-P_{h} u^{i}\right\|_{a}
$$

and

$$
\left|\lambda^{i, h}-\lambda_{i}\right| \leq C_{i}\left(\left(\lambda_{i, H}-\lambda_{i}\right)^{2}+\left\|u^{i}-u_{i, H}\right\|_{-a}^{2}+\left\|u^{i}-P_{h} u^{i}\right\|_{a}^{2}\right) .
$$


Consequently,

$$
\left\|u^{i}-u^{i, h}\right\|_{a} \leq C_{i}\left(\delta_{H}^{2}\left(\lambda_{i}\right)+\eta_{a}(H) \delta_{H}\left(\lambda_{i}\right)+\delta_{h}\left(\lambda_{i}\right)\right)
$$

and

$$
\left|\lambda^{i, h}-\lambda_{i}\right| \leq C_{i}\left(\delta_{H}^{4}\left(\lambda_{i}\right)+\eta_{a}^{2}(H) \delta_{H}^{2}\left(\lambda_{i}\right)+\delta_{h}^{2}\left(\lambda_{i}\right)\right) .
$$

Proof. From the identity

$$
a\left(P_{h} u^{i}-u^{i, h}, v\right)=\left(\lambda_{i}-\lambda_{i, H}\right) b\left(u_{i, H}, v\right)+\lambda_{i} b\left(u^{i}-u_{i, H}, v\right), \forall v \in S^{h},
$$

we immediately obtain (4.3). And (4.4) follows from (4.3) and Proposition 4.2 Finally, the last two conclusions are obtained from Proposition 3.1 .

\section{EXAMPLES}

The general two-grid discretization scheme presented in this paper can be applied to a large class of eigenvalue problems. In this section, as an illustration, we give two examples, one for a partial differential operator and another for an integral operator. Let $\Omega \subset R^{d}(d=1,2, \cdots)$ be a bounded domain and $T^{h}(\Omega)$, consisting of shape-regular simplices, be a mesh of $\Omega$ of size $h$.

5.1. Second order elliptic operators. Let $b(\cdot, \cdot)=(\cdot, \cdot)$ be the standard inner product of $L^{2}(\Omega)$, and let

$$
a(u, v)=\int_{\Omega} \sum_{i, j=1}^{d} a_{i j} \frac{\partial u}{\partial x_{i}} \frac{\partial v}{\partial x_{j}},
$$

satisfying $a_{i j} \in W^{1, \infty}(\Omega)$ and $\left(a_{i j}\right)$ is uniformly positive definite on $\Omega$.

Set $X_{a}=H_{0}^{1}(\Omega)$ and $X_{b}=L^{2}(\Omega)$. Define $S^{h}(\Omega)$ to be a space of continuous functions on $\Omega$ and vanishing at the boundary $\partial \Omega$, such that for $v \in S^{h}(\Omega), v$ restricted to each $\tau$ is a polynomial of total degree $\leq r$, namely

$$
S^{h}(\Omega)=\left\{v \in C(\bar{\Omega}) \cap H_{0}^{1}(\Omega):\left.v\right|_{\tau} \in P_{\tau}^{r}, \forall \tau \in T^{h}(\Omega)\right\},
$$

where $P_{\tau}^{r}$ is the space of polynomials of degree not greater than a positive integer $r$.

We assume that $M\left(\lambda_{i}\right) \subset H^{r+1}(\Omega)$. If $\Omega$ is polygonal and convex, then

$$
\eta_{a}(h)=O(h), \delta_{h}\left(\lambda_{i}\right) \leq C_{i} h^{r} .
$$

Hence

$$
\left\|u^{i}-u^{i, h}\right\|_{a} \leq C_{i}\left(H^{r+1}+h^{r}\right)
$$

and

$$
\left|\lambda^{i, h}-\lambda_{i}\right| \leq C_{i}\left(H^{2 r+2}+h^{2 r}\right) .
$$

If $\Omega$ has $C^{1}$-smooth boundary, then better estimates can be obtained for Neumann boundary value problems (using $H^{1}(\Omega) / R^{1}$, boundary elements fitting $\partial \Omega$ exactly and $r>1)$ :

$$
\eta_{a}(h)=O\left(h^{2}\right), \delta_{h}\left(\lambda_{i}\right) \leq C_{i} h^{r} .
$$

Hence

$$
\left\|u^{i}-u^{i, h}\right\|_{a} \leq C_{i}\left(H^{\min \{r+2,2 r\}}+h^{r}\right)
$$

and

$$
\left|\lambda^{i, h}-\lambda_{i}\right| \leq C_{i}\left(H^{2 \min \{r+2,2 r\}}+h^{2 r}\right) .
$$




\subsection{Fredholm integral operators. Let}

$$
(K w)(x)=\int_{\Omega} k(x, y) w(y) d y
$$

be the symmetric and positive definite Fredholm integral operator on $L^{2}(\Omega)$, where $k(x, y) \in C^{r+1}(\Omega \times \Omega)$. Set $a(\cdot, \cdot)=(\cdot, \cdot)$, the standard inner product of $L^{2}(\Omega), b(\cdot, \cdot)=(K \cdot, \cdot), X_{a}=L^{2}(\Omega)$ and $X_{b}=$ the completion of $X_{a}$ with respect to $\|\cdot\|_{b}$.

Define $S^{h}(\Omega)$ to be a space of continuous functions on $\Omega$ such that for $v \in$ $S^{h}(\Omega), v$ restricted to each $\tau$ is a polynomial of total degree $\leq r$, namely

$$
S^{h}(\Omega)=\left\{v \in C(\bar{\Omega}):\left.v\right|_{\tau} \in P_{\tau}^{r}, \forall \tau \in T^{h}(\Omega)\right\} .
$$

In this case, we have

$$
\eta_{a}(h)=O\left(h^{r+1}\right), \delta_{h}\left(\lambda_{i}\right) \leq C_{i} h^{r+1},
$$

and hence

$$
\left\|u^{i}-u^{i, h}\right\|_{a} \leq C_{i}\left(H^{2 r+2}+h^{r+1}\right)
$$

and

$$
\left|\lambda^{i, h}-\lambda_{i}\right| \leq C_{i}\left(H^{4 r+4}+h^{2 r+2}\right)
$$

\section{Nonselfadjoint PROBlems}

The approaches presented in the above can be applied to nonselfadjoint eigenvalue problems. We assume that $b(\cdot, \cdot)$ is symmetric but $a(\cdot, \cdot)$ may not be symmetric; then the following property of eigenvalue and eigenvector approximation can also be established for nonselfadjoint cases.

Proposition 6.1. Let $(\lambda, u)$ be an eigenvalue pair of (3.1). For any $w \in X_{a} \backslash\{0\}$,

$$
\begin{aligned}
\frac{a(w, w)}{b(w, w)}-\lambda= & \frac{a(w-u, w-u)}{b(w, w)}-\lambda \frac{b(w-u, w-u)}{b(w, w)} \\
& +\frac{a(w-u, u)-a(u, w-u)}{b(w, w)} .
\end{aligned}
$$

Note that, for instance,

$$
|a(w-u, u)-a(u, w-u)| \lesssim\|w-u\|_{1-r}
$$

and $\|w-u\|_{1-r} \ll\|w-u\|_{1}$, when $r>1$ and $a(\cdot, \cdot)$ is the bilinear form derived from a general elliptic problem of second order.

Hence, in this case, our approach will work well. In fact, other techniques for dealing with nonselfadjoint problems can also be employed, cf. [16].

\section{NumericAl EXPERIMENTS}

For illustration, in this section, we report some simple numerical experiments for a second order elliptic operator.

We consider the following eigenvalue problem:

$$
\begin{aligned}
-\Delta u & =\lambda u, & \text { in } \Omega, \\
u & =0, & \text { on } \partial \Omega,
\end{aligned}
$$




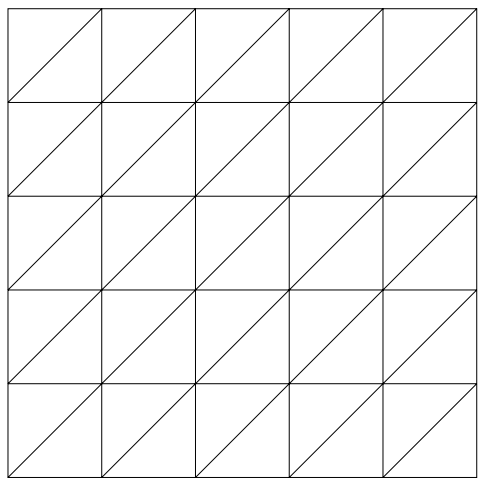

Figure 2. A triangulation

where $\Omega \subset R^{2}$ is the unit square domain $\Omega=(0,1) \times(0,1)$. The eigenvalues of (17.1) are easily seen to be given by

$$
\lambda_{k, l}=\left(k^{2}+l^{2}\right) \pi^{2},
$$

and are associated with the eigenvectors

$$
u_{k, l}=\sin (k \pi(x-1)) \sin (l \pi(y-1)), k, l=1,2, \cdots .
$$

Set a uniform triangulation $T^{h}(\Omega)=\{\tau\}$ (see Figure 21) and piecewise linear finite element space as follows:

$$
S^{h}(\Omega)=\left\{v \in H_{0}^{1}(\Omega):\left.v\right|_{\tau} \text { is linear, } \forall \tau \in T^{h}(\Omega)\right\} .
$$

Now we apply the algorithm to solve the problem (7.1) using the fine meshes of sizes $h=2^{-j}(j=4,6,8,10)$ and corresponding coarse meshes of size $H=\sqrt{h}$, and apply the algorithm as described in the introduction.

If $\lambda_{H}$ is the first eigenvalue of the problem, then by Theorem 4.3, we have

$$
\left\|\nabla\left(u_{h}-u^{h}\right)\right\|_{L^{2}}=\mathcal{O}\left(H^{2}\right) \approx c h \text { and }\left|\lambda^{h}-\lambda_{h}\right|=\mathcal{O}\left(H^{4}\right) \approx c h^{2},
$$

where $\left(\lambda_{h}, u_{h}\right)$ is the standard first finite element eigenpair on $T^{h}$.

The results shown in Table 1 are consistent with the above estimates.

TABLE 1. A two-grid algorithm for elliptic eigenvalue problems

\begin{tabular}{|c|c|c|c|c|}
\hline$h$ & $\left\|\nabla\left(u_{h}-u^{h}\right)\right\|_{L^{2}}$ & $\frac{\left\|\nabla\left(u_{4 h}-u^{4 h}\right)\right\|_{L^{2}}}{\left\|\nabla\left(u_{h}-u^{h}\right)\right\|_{L^{2}}}$ & $\left|\lambda_{h}-\lambda^{h}\right|$ & $\frac{\left|\lambda_{4 h}-\lambda^{4 h}\right|}{\left|\lambda_{h}-\lambda^{h}\right|}$ \\
\hline $2^{-4}$ & $0.7401 \mathrm{E}-01$ & & $0.1255 \mathrm{E}-01$ & \\
$2^{-6}$ & $0.2026 \mathrm{E}-01$ & 3.6527 & $0.9028 \mathrm{E}-03$ & 13.8980 \\
$2^{-8}$ & $0.5183 \mathrm{E}-02$ & 3.9090 & $0.5997 \mathrm{E}-04$ & 15.0572 \\
$2^{-10}$ & $0.1303 \mathrm{E}-02$ & 3.9767 & $0.3811 \mathrm{E}-05$ & 15.7322 \\
\hline
\end{tabular}




\section{ACKNOWLEDGMENT}

The authors wish to thank H. Kim for his assistance with numerical experiments.

\section{REFERENCES}

1. Adams R.A.(1975): Sobolev Spaces, Academic Press, New York. MR 56:9247

2. Axelsson, O. and Layton, W.(1996): A two-level discretization of nonlinear boundary value problems, SIAM J. Numer. Anal., 33, 2359-2374. MR 98c:65181.

3. Babuska, I. and Osborn, J.E.(1989): Finite element-Galerkin approximation of the eigenvalues and eigenvectors of selfadjoint problems, Math. Comp., 52, 275-297. MR 89k:65132

4. Babuska, I. and Osborn, J.E.(1991): Eigenvalue problems, Handbook of Numerical Analysis, Vol. II, Finite Element Methods (Part 1) (P.G. Ciarlet and J.L. Lions, eds.), Elsevier, 641-792. MR 92f:65001

5. Chatelin, F.(1983): Spectral Approximations of Linear Operators, Academic Press, New York. MR 86d:65071

6. Ciarlet, P.G. and Lions J.L.(1991): Handbook of Numerical Analysis, Vol.II, Finite Element Methods (Part I), North-Holland. MR 92f:65001

7. Dawson, C.N. and Wheeler, M.F.(1994): Two-grid methods for mixed finite element approximations of nonlinear parabolic equations, Contemp. Math., 180, 191-203. MR 95j:65117

8. Dawson, C.N., Wheeler, M.F. and Woodward, C.S.(1998): A two-grid finite difference scheme for nonlinear parabolic equations, SIAM J. Numer. Anal.,35, 435-452. MR 99b:65097

9. Layton, W. and Lenferink, W.(1995): Two-level Picard and modified Picard methods for the Navier-Stokes equations, Appl. Math. Comp., 69, 263-274. MR 95m:65191

10. Lin, Q.(1979): Some problems concerning approximate solutions of operator equations, Acta Math. Sinica, 22, 219-230(Chinese). MR 80k:65056

11. Marion, M. and Xu, J.(1995): Error estimates on a new nonlinear Galerkin method based on two-grid finite elements, SIAM J. Numer. Anal., 32, 1170-1184. MR 96f:65136

12. Utnes, T.(1997): Two-grid finite element formulations of the incompressible Navier-Stokes equations, Comm. Numer. Methods Engrg., 34, 675-684. MR 98d:76110

13. Xu, J.(1992): A new class of iterative methods for nonselfadjoint or indefinite problems, SIAM J. Numer. Anal., 29, 303-319. MR 92k:65063

14. Xu, J.(1992): Iterative methods by space decomposition and subspace correction, SIAM Review, 34, 4, 581-613. MR 93k:65029

15. Xu, J.(1994): A novel two-grid method for semilinear equations, SIAM J. Sci. Comput., 15, 231-237. MR 94m:65178

16. Xu, J.(1996): Two-grid discretization techniques for linear and nonlinear PDEs, SIAM J. Numer. Anal., 33, 1759-1777. MR 97i:65169

17. Xu, J. and Zhou, A.(1998): Local and parallel finite element algorithms based on two-grid discretizations, Math. Comp.(to appear).

Center for Computational Mathematics and Applications, Department of Mathematics, Pennsylvania State University, University Park, Pennsylvania 16802

E-mail address: xu@math.psu.edu

Institute of Systems Science, Academia Sinica, Beijing 100080, China

E-mail address: azhou@bamboo.iss.ac.cn 